\title{
Estudio Termodinámico de la Remoción de Níquel y Cromo en Solución Acuosa usando Adsorbentes de Origen Agroindustrial
}

\author{
Erika B. Ruiz-Paternina(1), Ángel Villabona-Ortiz(1), Candelaria Tejada-Tovar ${ }^{(1)}$ y Rodrigo Ortega-Toro(2)* \\ (1) Facultad de Ingeniería, Programa de Ingeniería Química, Grupo de Investigación en Diseño de Procesos y \\ Aprovechamiento de Biomasa (IDAB), Universidad de Cartagena, Avenida del Consulado Calle 30 No. 48 - 152 , \\ Colombia (e-mail: erikbeatriz@gmail.com, avillabonao@unicartagena.edu.co; ctejadat@unicartagena.edu.co) \\ (2) Facultad de Ingeniería, Programa de Ingeniería de Alimentos, Grupo de Investigación Food Packaging and Shelf Life \\ (FP\&SL) Universidad de Cartagena, Avenida del Consulado Calle 30 No. 48 - 152, Colombia. \\ (e-mail: rortegap1@unicartagena.edu.co)
}

${ }^{*}$ Autor a quien debe ser dirigida la correspondencia

Recibido Dic. 17, 2018; Aceptado Mar. 3, 2019; Versión final May. 7, 2019, Publicado Dic. 2019

\begin{abstract}
Resumen
El objetivo del presente trabajo fue estudiar el proceso de adsorción en una columna de lecho empacado usando bio-adsorbentes obtenidos de subproductos agroindustriales (pulpa de almidón de plátano, pulpa de almidón de ñame y bagazo de palma aceitera) para la eliminación de $\mathrm{Cr}$ (VI) y Ni (II) en solución acuosa. Se analizó el efecto e incidencia estadística sobre los cambios en la temperatura y altura del lecho en el proceso de adsorción de los metales pesados en una columna empacada con las biomasas en estudio, la concentración de los metales se fijó en $100 \mathrm{ppm}$ y el caudal en $0,75 \mathrm{~mL} / \mathrm{s}$. A partir de las pruebas de adsorción hechas a las condiciones estudiadas y el análisis estadístico de las mismas se determinó que la temperatura y la altura del lecho afectan significativamente la adsorción de $\mathrm{Cr}(\mathrm{VI})$ en la torta de plátano, y la altura del lecho afecta la adsorción de $\mathrm{Ni}$ (II) en la torta de ñame y bagazo de palma.
\end{abstract}

\section{Thermodynamic Study of the Removal of Nickel and Chromium in Aqueous Solution using Adsorbents of Agro-Industrial Origin}

\begin{abstract}
The objective of the present work was to study the adsorption process in a packed bed column using bioadsorbents obtained from agro-industrial by-products (plantain starch pulp, yam starch pulp and palm oil bagasse) for the elimination of $\mathrm{Cr}(\mathrm{VI})$ and $\mathrm{Ni}$ (II) in aqueous solution. The effect and statistical incidence on the changes in the temperature and bed height in the adsorption process of heavy metals in a column packed with the biomasses under study, was analysed, the metal concentration was fixed at $100 \mathrm{ppm}$ and the flow in $0.75 \mathrm{~mL} / \mathrm{s}$. From the adsorption tests made to the conditions studied and the statistical analysis of them, it was determined that the temperature and the height of the bed significantly affect the adsorption of $\mathrm{Cr}(\mathrm{VI})$ in the plantain cake, and the height of the bed affects $\mathrm{Ni}$ (II) adsorption on yam cake and palm bagasse.
\end{abstract}

Keywords: adsorption; packed-bed column; metallic ions; agro-industrial by-products 


\section{INTRODUCCIÓN}

Es evidente que las fuentes de agua subterráneas han sido contaminadas con metales pesados, ya sea por actividades antropogénicas o factores naturales (Manirethan et al., 2018). Diversos investigadores han realizado estudios para reducir la presencia de estos contaminantes disueltos, aplicando procesos como la precipitación química, el intercambio iónico, procesos de membrana, electrodiálisis, fito-extracción, ultrafiltración y osmosis inversa (Choppala et al., 2012); estos presentan desventajas tales como la eliminación incompleta de los metales, alto costo de reactivos y operación, requisitos de energía y generación de lodos tóxicos (Tohdee et al., 2018). En este sentido, la adsorción es un método efectivo y económico para el tratamiento de aguas residuales, resaltando el uso de materiales residuales.

Hay muchos adsorbentes eficientes para la remoción de metales pesados, materiales naturales como el quitosano (Zhang et al., 2016), arcillas (Ghorbel-Abid y Trabelsi-Ayadi, 2015), zeolitas (Qiu et al., 2018), carbones activados (Aguayo-Villarreal et al., 2017). Respecto a los anteriores se destacan los residuos agrícolas, tales como cáscaras de yuca (Xie et al., 2017), papa (Asuquo y Martin, 2016), naranja (Guiza, 2017), cascarilla de arroz (Qu et al., 2018), entre otros, ya que son de bajo costo además de ser ideales como adsorbentes porque presentan propiedades de gran superficie, alta capacidad de adsorción, estabilidad mecánica, compatibilidad, fácil acceso, facilidad de regeneración, rentabilidad, bajo costo, procedimientos simples y altamente selectivos (Zhang et al., 2016). En el presente trabajo, se propone el uso de los residuos de la pulpa de almidón de ñame y de plátano, y bagazo de palma, como adsorbentes de $\mathrm{Cr}$ (VI) y Ni (II) en una columna de lecho empacado. Las variables estudiadas del proceso fueron la altura de lecho y la temperatura, se establecieron además los parámetros termodinámicos del proceso de adsorción de iones metálicos.

\section{MATERIAL Y MÉTODOS}

A continuación, se describe la metodología usada para preparar los biomateriales, las pruebas de adsorción en la columna de lecho empacado y los modelos matemáticos usados para describir su comportamiento.

\section{Preparación de los biomateriales}

El plátano, el ñame y el bagazo de palma se recolectaron en zonas rurales del departamento de Bolívar (Colombia). La pulpa residual de ñame y de plátano fue recuperada como producto de rechazo después de ser sometidas a la extracción del almidón, para lo cual estos fueron pelados, lavados con agua destilada, puestos en una solución de $\mathrm{NaOH}$ al $0,25 \%$ a $5 \stackrel{\circ}{ } \mathrm{C}$ durante $18 \mathrm{~h}$, y separados por filtración (Maniglia y TapiaBlácido, 2016). El bagazo de palma fue obtenido como producto de rechazo de la extracción de aceite de palma; éste se sometió a tres lavados para remover la mayor cantidad de aceite retenido en la fibra, y secado al sol, y se separó de cascarillas y semillas. La clasificación de los tamaños de partícula para todas las biomasas se realizó en una tamizadora tipo Shaker a través de una serie de tamices de: $2 \mathrm{~mm}$ y $1 \mathrm{~mm}$.

\section{Pruebas de adsorción en columna de lecho empacado}

Se prepararon soluciones madre de $\mathrm{Cr}$ (VI) y Ni (II) a $1000 \mathrm{ppm}$ cada una, usando como reactivos base dicromato de potasio $\left(\mathrm{K}_{2} \mathrm{Cr}_{2} \mathrm{O}_{7}\right)$ y sulfato de níquel $\left(\mathrm{NiSO}_{4}\right)$. Los experimentos de adsorción se llevaron a cabo en una columna de acrílico que contenía las soluciones metálicas fluyendo hacia la columna con un caudal de $0,750 \mathrm{~mL} / \mathrm{s}$. Las variables estudiadas fueron altura de lecho y temperatura. La concentración final de los iones en solución fue tomada en la salida inferior de la columna, y se determinaron mediante espectrofotometría UV-VIS para Cr (VI) y espectrofotometría de absorción atómica para Ni (II).

La eficiencia de remoción $\left(\mathrm{R}_{\mathrm{E}}\right)$ se determinó usando la ecuación (1).

$$
\text { (\%) } R_{E}=\left[\frac{C_{O}-C_{T}}{C_{O}} \times 100\right]
$$

Donde $C_{T}$ es la concentración final del ion de $\mathrm{Cr}(\mathrm{VI})$ o $\mathrm{Ni}(\mathrm{II})$ a la salida de la columna en ppm y $C_{0}$ la concentración inicial del metal en ppm, la concentración usada en todos los casos fue 100 ppm.

Los resultados fueron procesados con el Software Statgraphics Centurion XVI determinando las variables significativas del proceso mediante análisis de varianza ANOVA y las condiciones óptimas de adsorción para cada sistema metal/adsorbente, con las cuales se hizo la curva de ruptura y el ajuste a modelos. Los Modelos usados para tal ajuste se describen a continuación. 
Modelo de Thomas. Este se muestra en la ecuación (2).

$$
\frac{C_{o}}{C}=\frac{1}{1+\operatorname{EXP}\left[\frac{K_{t h}}{Q}\left(q_{o} X-C_{o} V\right]\right.}
$$

Donde $K_{\text {th }}$, es la constante de Thomas $\left(\mathrm{mL} / \mathrm{min}^{-1} \mathrm{mg}^{-1}\right) ; q_{o}$, es la concentración máxima de soluto en la fase solida $(\mathrm{mg} / \mathrm{g}) ; X$, es la cantidad de adsorbente en la columna $(\mathrm{g}), Q$ es la velocidad de flujo $(\mathrm{mL} / \mathrm{min})$ y $V(\mathrm{~L})$ es el volumen del efluente en el tiempo de operación.

Modelo Yoon-Nelson. Este modelo relaciona el parámetro adimensional $C / C_{0}$ con el tiempo de operación mediante dos parámetros $K_{Y N}$ y $\tau$ correspondiente a la constante de proporcionalidad en min-1 y al tiempo para retener el $50 \%$ del adsorbato inicial respectivamente. Este se muestra en la ecuación (3).

$$
\frac{C}{C_{o}}=\frac{\operatorname{Exp}\left(K_{Y N} t-\tau K_{Y N}\right)}{1+\operatorname{Exp}\left(K_{Y N} t-\tau K_{Y N}\right)}
$$

Modelo Dosis de respuesta. El modelo DR puede ser representado por la ecuación (4).

$$
\frac{C}{C_{o}}=1-\frac{1}{1+\left[\frac{C_{O} * Q t}{q_{o} X}\right]^{a}}
$$

Donde a es la constante del modelo; $q_{0}$, concentración máxima de soluto en la fase sólida $(\mathrm{mg} / \mathrm{g}), X$, es la cantidad de adsorbente en la columna (g) y $Q$ es la velocidad de flujo $(\mathrm{mL} / \mathrm{min})$.

La capacidad experimental de adsorción de la columna se determinó usando la ecuación (5).

$$
Q_{\text {total }}=\frac{Q}{1000 m} \int_{0}^{t s} C t-C o d t
$$

Donde $Q_{\text {total }}$ es la concentración del ion de $\mathrm{Cr}(\mathrm{VI})$ o $\mathrm{Ni}$ (II) en el adsorbente $(\mathrm{mg} / \mathrm{g}) ; C_{0}$ es la concentración del ion o los iones en la alimentación en solución acuosa; $C_{t}$ es la concentración de salida de los iones estudiados en la solución acuosa ( $\mathrm{mg} / \mathrm{L})$; ts es el tiempo que demora el adsorbente en convertir la concentración inicial del ion estudiado en la concentración final ( $\mathrm{min}$ ); $Q$ es el caudal en $\mathrm{mL} / \mathrm{min}$ y $\mathrm{m}$ es la masa de la torta empacada en la columna $(\mathrm{g})$.

Los parámetros termodinámicos se determinaron usando el método gráfico basado en la ecuación de Van't Hoff, así se estimaron: el cambio en la energía libre estándar de Gibbs $(\Delta \mathrm{G})$ utilizando las ecuaciones $(6)$ y (7), la entalpia $(\Delta \mathrm{H})$ y entropía estándar $(\Delta S)$ utilizando la ecuación (8) en su forma gráfica.

$$
\begin{aligned}
& \Delta G=\Delta H-T \Delta S \\
& \Delta G=-R T * \ln k_{c} \\
& \ln K_{c}=\frac{-\Delta H}{R * T}+\frac{\Delta S}{R}
\end{aligned}
$$

Donde $K c=C a c / C s e ; C a c$ es la concentración del adsorbato en el equilibrio contenido en la superficie del adsorbente; Cse es la concentración en solución en el equilibrio y $R$ es la constante universal de los gases $(8,314 \mathrm{~J} / \mathrm{mol} . \mathrm{K})$. Se realizaron pruebas de adsorción del metal a una altura de lecho de $6,5 \mathrm{~cm}$ a $30,55, \mathrm{y}$ $76{ }^{\circ} \mathrm{C}$ y concentración de metal de 100 ppm.

\section{RESULTADOS Y DISCUSIÓN}

De la Tabla 1 se observa que la altura de lecho y temperatura tienen una incidencia significativa sobre el proceso de adsorción de $\mathrm{Cr}(\mathrm{VI})$ con la pulpa residual de almidón de plátano, ya que el valor-P menor que 0,05 , así lo indica, mientras que tales variables no son significativas para los otros procesos. Del análisis de varianza mostrado en la Tabla 2 se observa que la altura de lecho y temperatura tienen una incidencia significativa sobre el proceso de adsorción de Ni (II) con el bagazo de palma y pulpa residual de almidón de 
ñame. Mostrando así que los procesos donde hay incidencia de las variables en la remoción del metal podría deberse en efecto a la interacción de unión adsorbato-adsorbente o formación de nuevos sitios efectivos para la adsorción

Tabla 1: Análisis de varianza para la adsorción de $\mathrm{Cr}(\mathrm{VI})$

\begin{tabular}{|c|c|c|c|c|c|c|c|c|c|}
\hline Biomasa & \multicolumn{3}{|c|}{ Bagazo de palma name } & \multicolumn{3}{c|}{ Torta residual de almidón de ñdual de almidón de plátano } \\
\hline Fuente & $\begin{array}{c}\text { Suma de } \\
\text { Cuadrados }\end{array}$ & Razón-F & Valor-P & $\begin{array}{c}\text { Suma de } \\
\text { Cuadrados }\end{array}$ & Razón-F & Valor-P & $\begin{array}{c}\text { Suma de } \\
\text { Cuadrados }\end{array}$ & Razón-F & Valor-P \\
\hline A: Temperatura & 0,001 & 0,230 & 0,653 & $1,23 \mathrm{E} 19$ & 1,33 & 0,312 & 801,66 & 13,71 & $\mathbf{0 , 0 3 0}$ \\
\hline B: Altura de lecho & 0,001 & 1,50 & 0,288 & $1,23 \mathrm{E} 19$ & 1,33 & 0,312 & 819,53 & 14,02 & $\mathbf{0 , 0 3 0}$ \\
\hline A & 0,001 & 0,370 & 0,575 & $1,76 \mathrm{E} 18$ & 0,190 & 0,685 & 64,62 & 1,11 & 0,370 \\
\hline AB & 0,001 & 0,470 & 0,531 & $2,47 \mathrm{E} 19$ & 2,67 & 0,178 & 44,55 & 0,76 & 0,450 \\
\hline BB & 0,001 & 0,010 & 0,909 & $1,76 \mathrm{E} 18$ & 0,190 & 0,685 & 166,84 & 2,85 & 0,190 \\
\hline Error total & 0,002 & & & $3,70 \mathrm{E} 19$ & & & 175,38 & & \\
\hline Total (corr.) & 0,003 & & & $8,86 \mathrm{E} 19$ & & & 2008,04 & & \\
\hline
\end{tabular}

Tabla 2: Análisis de varianza para la adsorción de Ni (II)

\begin{tabular}{|c|c|c|c|c|c|c|c|c|c|}
\hline Biomasa & \multicolumn{4}{|c|}{ Bagazo de palma } & \multicolumn{2}{c|}{ Torta residual de almidón de ñame } & \multicolumn{2}{c|}{ Torta residual de almidón de plátano } \\
\hline Fuente & $\begin{array}{c}\text { Suma de } \\
\text { Cuadrados }\end{array}$ & Razón-F & Valor-P & $\begin{array}{c}\text { Suma de } \\
\text { Cuadrados }\end{array}$ & Razón-F & Valor-P & $\begin{array}{c}\text { Suma de } \\
\text { Cuadrados }\end{array}$ & Razón-F & Valor-P \\
\hline A: Temperatura & 3,74 & 0,640 & 0,467 & 10,62 & 1,45 & 0,295 & 0,788 & 0,360 & 0,590 \\
\hline B: Altura de lecho & 115,03 & 19,79 & 0,011 & 126,84 & 17,32 & 0,014 & 3,19 & 1,450 & 0,310 \\
\hline AA & 26,23 & 4,51 & 0,101 & 7,225 & 0,990 & 0,377 & 0,006 & 0,000 & 0,960 \\
\hline AB & 1,88 & 0,320 & 0,600 & 72,40 & 9,880 & 0,035 & 0,438 & 0,200 & 0,680 \\
\hline BB & 4,37 & 0,750 & 0,435 & 114,40 & 15,62 & 0,017 & 0,398 & 0,180 & 0,700 \\
\hline Error total & 23,25 & & & 29,30 & & & 6,60 & & \\
\hline Total (corr.) & 170,14 & & & 357,96 & & & 11,59 & & \\
\hline
\end{tabular}

En la Figura 1 se muestra el comportamiento con respecto a la temperatura y la altura de lecho en la adsorción de $\mathrm{Cr}(\mathrm{VI})$. Se observa un aumento del porcentaje de remoción por el incremento de la temperatura, esto puede obedecer a una mayor tasa de difusión de iones metálicos en los mesoporos de la pulpa residual debido a la formación de enlaces con los grupos funcionales mejorando la tasa de difusión intra-partícular. El comportamiento de la altura de lecho se puede explicar con el incremento en el tiempo de penetración de la solución, lo que representa un mayor tiempo de residencia, en consecuencia, el metal tiene más tiempo para difundirse en la fase sólida (Mishra et al., 2016).
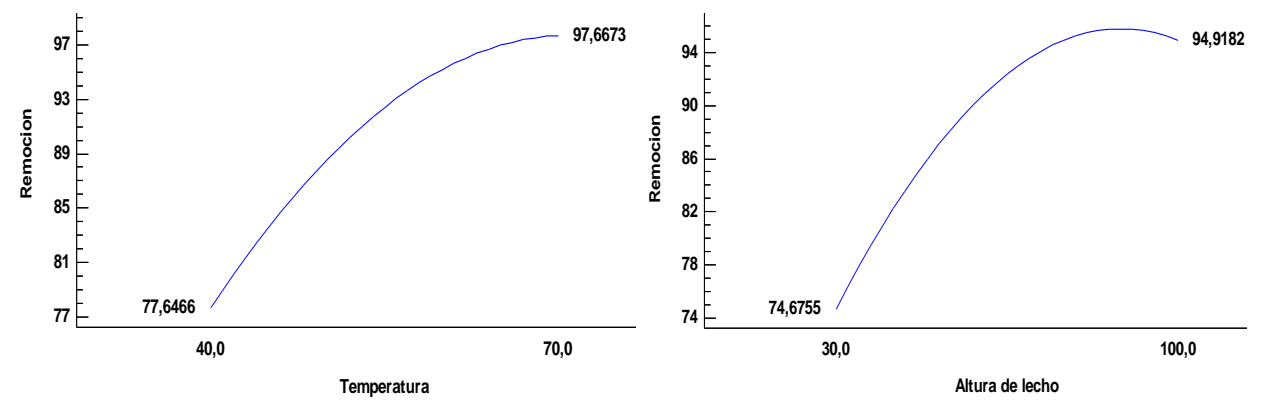

Fig. 1: Efecto de la temperatura (izquierda) y altura de lecho (derecha) en la remoción de $\mathrm{Cr}(\mathrm{VI})$ sobre la torta residual de almidón de plátano. Concentración inicial $100 \mathrm{ppm}, \mathrm{Q}: 0,75 \mathrm{~mL} / \mathrm{s}$, tamaño de partícula: $1 \mathrm{~mm}, \mathrm{pH}: 2$.

La Figura 2 muestra el comportamiento del \% de remoción de Ni (II) respecto a la altura del lecho usando bagazo de palma y torta residual de almidón de ñame.
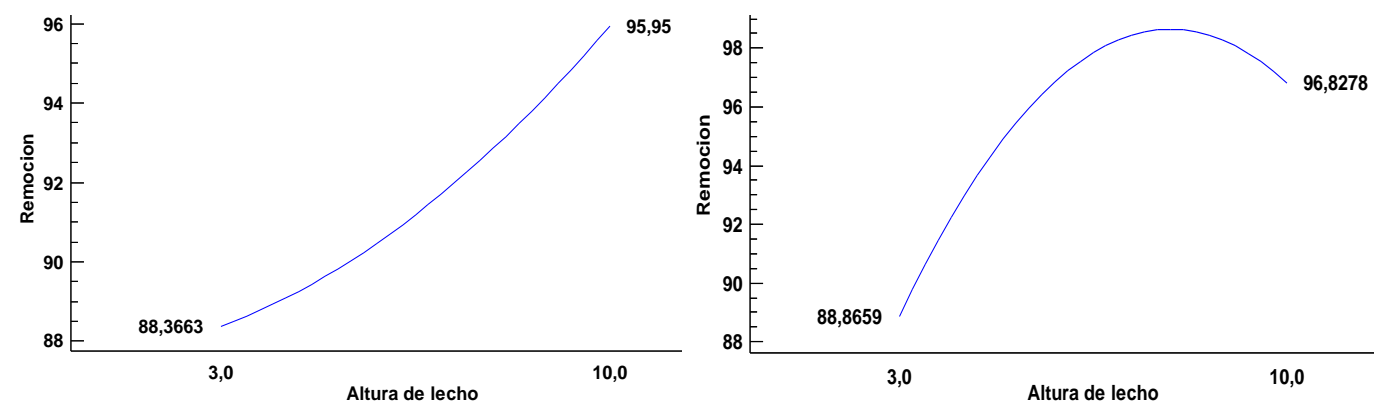

Fig. 2: Efecto de la altura de lecho en la remoción de Ni (II) sobre el bagazo de palma (izquierda) y la torta residual de almidón de ñame (derecha). Concentración inicial 100 ppm, Q: 0,75 mL/s, tamaño de partícula: 1mm, pH:6. 
De la Figura 2 se puede afirmar que a mayor cantidad de adsorbente en la columna, hay más sitios de unión disponibles, obteniendo una zona de transferencia de masa ampliada. Para profundidades de lecho más pequeñas, los fenómenos de dispersión axial predominan en el proceso de transferencia de masa lo que conduce a una reducción de la difusividad de los iones metálicos. En consecuencia, los iones metálicos no tienen el tiempo suficiente para difundir en el adsorbente, dejando antes la columna. Se observa que, en el caso de la torta de ñame, este alcanza un porcentaje de remoción máximo y luego disminuye, lo que indica un agotamiento del material o posibles cambios debido a la interacción que pudiese tener esta con la temperatura.

\section{Curvas de ruptura para la adsorción de $\mathrm{Cr}$ (VI) y Ni (II)}

Las curvas de ruptura fueron trabajadas con las condiciones óptimas para cada proceso. Para la adsorción de $\mathrm{Cr}(\mathrm{VI})$ con bagazo de palma, pulpa residual de ñame y plátano, fueron 1,55, 1,5 y 8,14 cm de altura de lecho y 76,33 , y $68^{\circ} \mathrm{C}$, respectivamente. Para el $\mathrm{Ni}$ (II) fueron $11,4 \mathrm{~cm}$ para el bagazo y la pulpa de plátano, $9 \mathrm{~cm}$ para la de ñame, y 76,43 y $33^{\circ} \mathrm{C}$ como condición óptima del bagazo de palma, pulpa residual de ñame y plátano, respectivamente. Los ajustes y parámetros para $\mathrm{Cr}(\mathrm{VI})$ se muestran en la Figura 3 y Tabla 3 , respectivamente.

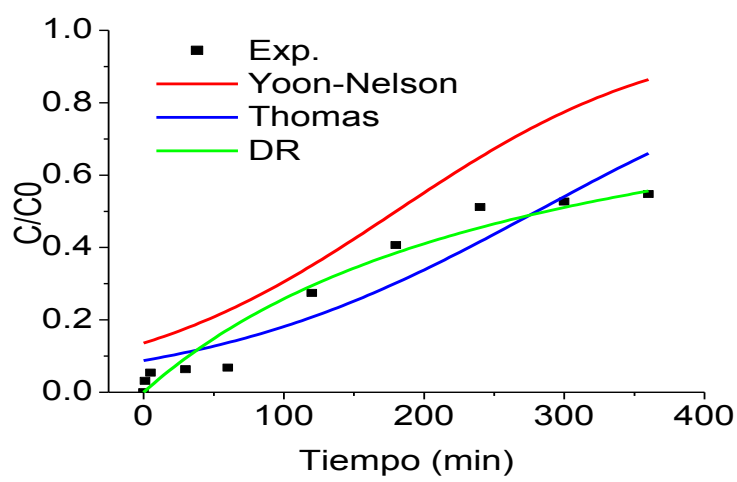

A

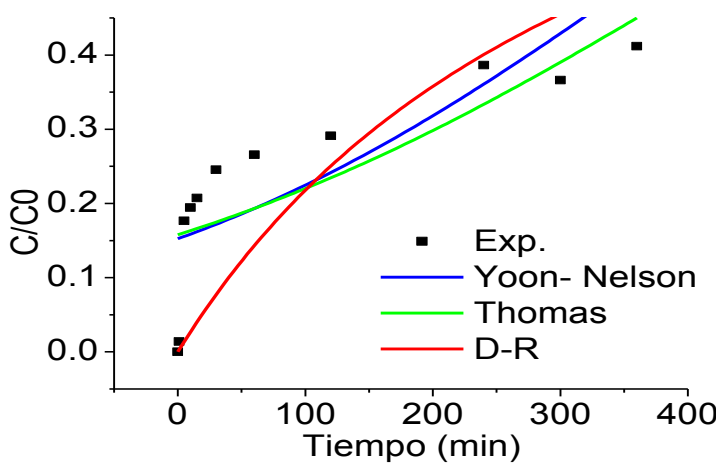

B

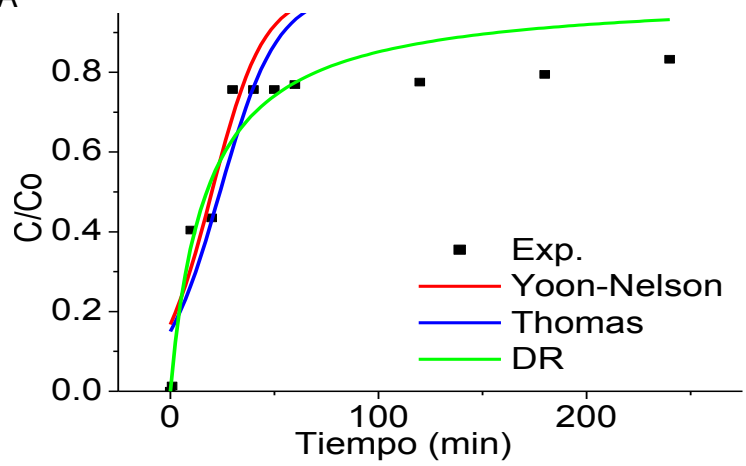

C

Fig. 3: Ajuste de modelos de curva de ruptura en la adsorción de $\mathrm{Cr}(\mathrm{VI})$ sobre el bagazo de palma (a), pulpa residual de almidón de plátano (b) y pulpa residual de almidón de ñame (c).

Tabla 3: Parámetros de ajuste para la adsorción de Cr (VI)

\begin{tabular}{|c|c|c|c|c|c|c|}
\hline \multirow{3}{*}{$\begin{array}{l}\text { Biomasa } \\
\text { Capacidad } \\
\text { experimental }\end{array}$} & \multicolumn{2}{|c|}{ Torta residual de almidón de ñame } & \multicolumn{2}{|c|}{$\begin{array}{c}\text { Torta residual de almidón } \\
\text { de plátano }\end{array}$} & \multicolumn{2}{|c|}{ Bagazo de palma } \\
\hline & Parámetros & $r^{2}$ & Parámetros & $r^{2}$ & Parámetros & $r^{2}$ \\
\hline & \multicolumn{2}{|l|}{$28,01 \mathrm{mg} / \mathrm{g}$} & \multicolumn{2}{|c|}{$18,25 \mathrm{mg} / \mathrm{g}$} & \multicolumn{2}{|c|}{$49,58 \mathrm{mg} / \mathrm{g}$} \\
\hline Modelo de Thomas & $\begin{array}{l}k_{T h}: 0,724 \mathrm{~mL} / \mathrm{min} \cdot \mathrm{mg} \\
q_{T h}: 8,97 \mathrm{mg} / \mathrm{g}\end{array}$ & 0,725 & $\begin{array}{c}k_{T h}: 0,041 \\
q_{T h}: 30,68\end{array}$ & 0,599 & $\begin{array}{l}k_{T h}: 0,072 \\
q_{T h}: 66,83\end{array}$ & 0,714 \\
\hline $\begin{array}{l}\text { Modelo Dosis } \\
\text { Respuesta }\end{array}$ & $\begin{array}{l}\mathrm{q}_{\mathrm{D}-\mathrm{R}}: 0,793 \\
\mathrm{a}: 1,94\end{array}$ & 0,926 & $\begin{array}{c}q_{D-R}: 0,203 \\
a: 4,08\end{array}$ & 0,139 & $\begin{array}{c}q_{D-R}: 0,180 \\
a: 6,19\end{array}$ & 0,879 \\
\hline Modelo Yoon-Nelson & $\mathrm{k}_{\mathrm{Y}-\mathrm{N}: 0,0801 / \mathrm{min}}$ & 0,734 & $\mathrm{k}_{\mathrm{Y}-\mathrm{N}:} 0,047$ & 0,612 & $\mathrm{k}_{\mathrm{Y}-\mathrm{N}: 0,009}$ & 0,150 \\
\hline
\end{tabular}

De los modelos estudiados, se observa que el de Dosis-Respuesta proporciona mejor ajuste a las curvas de avance mostrando buena concordancia con los valores experimentales. Para la pulpa residual de almidón de plátano ningún modelo ajusta el proceso, con lo cual se describen moderadamente los datos. La capacidad de adsorción experimental de $\mathrm{Cr}(\mathrm{VI})$ de la columna expresada en mg de $\mathrm{Cr}(\mathrm{VI})$ removido por gramo de adsorbente, mostró ser mayor para el bagazo de palma; esto se atribuye a la textura altamente porosa y la superficie fibrosa típica de los materiales lignocelulósicos (Pradhan et al., 2018). Otros autores encontraron capacidades de adsorción similares; usando residuos de calabaza blanca se encontró que era de 18,7 mg/g, 
además revelaron que el rendimiento de la columna se vio significativamente afectado por la altura del lecho, la tasa de flujo y la concentración inicial de ion metálico. El aumento de la altura del lecho resultó en una mayor tasa de biosorción (Sreenivas et al., 2014).

En la Figura 4 y Tabla 4 se muestran los ajustes y parámetros calculados para la adsorción de Ni (II).

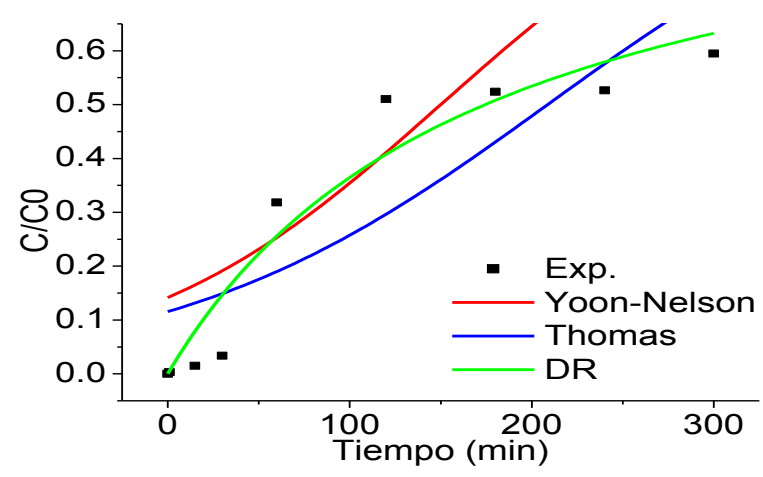

A

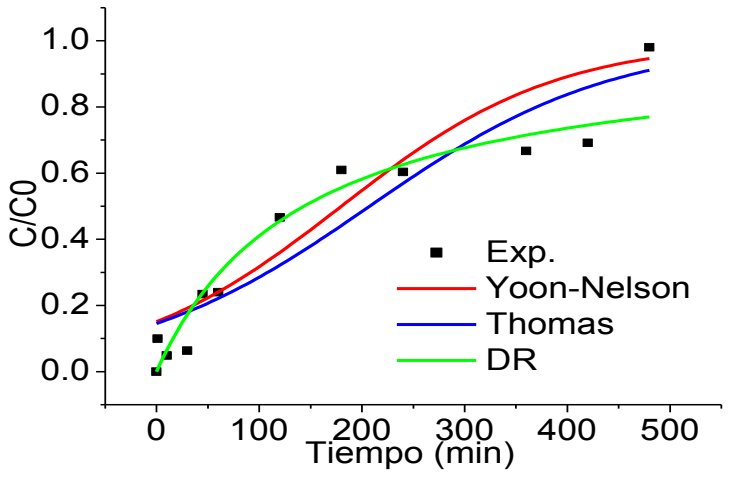

B

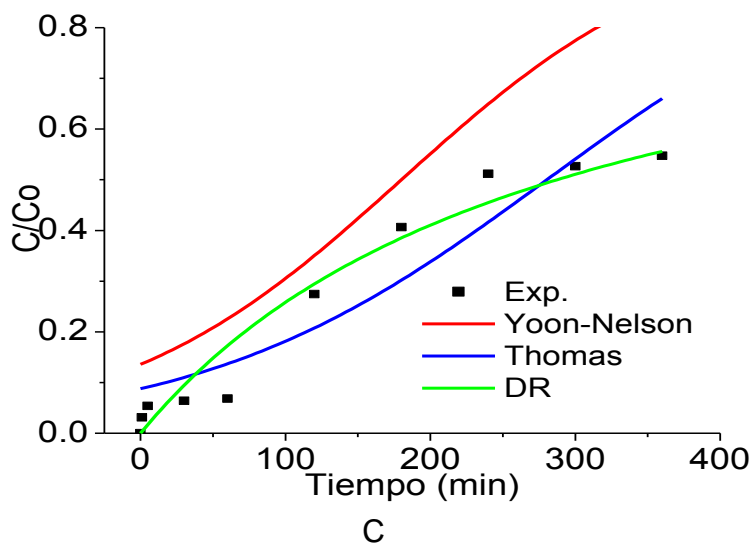

Fig. 4: Ajuste de modelos de curva de ruptura en la adsorción de Ni (II) sobre el bagazo de palma (a), torta residual de almidón de plátano (b) y torta residual de almidón de ñame (c)

Se establece nuevamente el modelo de Dosis Respuesta el de mejor ajuste. Sin embargo, es difícil relacionar el parámetro empírico "a" con las condiciones experimentales estudiadas en este tipo de sistemas, por lo que resulta prácticamente imposible la realización de un cambio de escala del sistema con este modelo. A diferencia de la adsorción de $\mathrm{Cr}(\mathrm{VI})$, las curvas de avance realizadas para la adsorción de Ni (II) a pesar de tener un comportamiento similar en la adsorción pronunciada en los primeros instantes de contacto con la biomasa adsorbente, muestra un punto de quiebre cercano a las tres horas de operación, debido a la ocupación gradual de los sitios activos disponibles (Abdolali et al., 2017) mostrando una tendencia en la disminución de la capacidad de adsorción más pronunciada a la saturación completa del adsorbente durante las ocho horas de operación continua indicando que para la adsorción de Ni (II) la torta residual de almidón de plátano tiene mayor vida útil en el proceso de remoción.

Tabla 4: Parámetros de ajuste para la adsorción de Ni (II)

\begin{tabular}{|c|c|c|c|c|c|c|}
\hline \multirow{3}{*}{$\begin{array}{c}\text { Biomasa } \\
\begin{array}{c}\text { Capacidad } \\
\text { experimental }\end{array}\end{array}$} & \multicolumn{2}{|c|}{ Torta residual de almidón de ñame } & \multicolumn{2}{|c|}{$\begin{array}{l}\text { Torta residual de almidón de } \\
\text { plátano }\end{array}$} & \multicolumn{2}{|c|}{ Bagazo de palma } \\
\hline & Parámetros & $r^{2}$ & Parámetros & $r^{2}$ & Parámetros & $r^{2}$ \\
\hline & \multicolumn{2}{|l|}{$28,01 \mathrm{mg} / \mathrm{g}$} & \multicolumn{2}{|c|}{$22,08 \mathrm{mg} / \mathrm{g}$} & \multicolumn{2}{|c|}{$26,29 \mathrm{mg} / \mathrm{g}$} \\
\hline $\begin{array}{l}\text { Modelo de } \\
\text { Thomas }\end{array}$ & $\begin{array}{c}k_{T h}: 0,083 \mathrm{~mL} / \mathrm{min} . \mathrm{mg} \\
q_{T h}: 33,21 \mathrm{mg} / \mathrm{g}\end{array}$ & 0,866 & $\begin{array}{l}k_{T h}: 0,085 \\
q_{T h}: 20,75\end{array}$ & 0,855 & $\begin{array}{l}k_{T h}: 0,097 \\
q_{T h}: 32,38\end{array}$ & 0,715 \\
\hline $\begin{array}{l}\text { Modelo Dosis } \\
\text { Respuesta }\end{array}$ & $\begin{array}{c}\mathrm{q}_{\mathrm{D}-\mathrm{R}}: 2,31 \mathrm{mg} / \mathrm{g} \\
\mathrm{a}: 1,61\end{array}$ & 0,996 & $\begin{array}{l}\text { qD-R }: 1,58 \\
a: 2,45\end{array}$ & 0,937 & $\begin{array}{c}\text { qD-R }: 0,570 \\
a: 2,37\end{array}$ & 0,927 \\
\hline $\begin{array}{l}\text { Modelo Yoon- } \\
\text { Nelson }\end{array}$ & $\mathrm{k}_{\mathrm{Y}-\mathrm{N}}: 0,0101 / \mathrm{min}$ & 0,431 & $k_{Y-N}: 0,009$ & 0,856 & $\mathrm{k}_{\text {Y-N }}: 0,012$ & 0,601 \\
\hline
\end{tabular}

Teniendo en cuenta las aplicaciones industriales del proceso de adsorción, la identificación del tiempo de avance es de vital importancia, ya que define el límite operacional de la columna, este punto de avance se define como el intervalo de tiempo en el que la concentración de salida es más alta que un umbral establecido, aunque en el presente estudio no se alcanzó tal saturación, se establece que en el tiempo de estudio todas las biomasas pueden ser usadas (Ramos et al., 2017). La máxima capacidad de adsorción se alcanzó con la torta residual de ñame, reportando resultados similares a otros autores: 29,93 mg/g con cáscaras de maracuyá 
(Chao et al., 2014), 23,47 mg/g con aserrín magnetizado (Kapur y Mondal, 2016), 19,36 mg/g con hojas nativa de teca (Vilvanathan y Shanthakumar, 2017) y $60,39 \mathrm{mg} / \mathrm{g}$ con bagazo de caña de azúcar carboxilado (Pedrosa et al., 2018), sin embargo este último estudio fue llevado a cabo a concentraciones inicial de metal superiores a las del presente estudio, por cuanto había más disponibilidad para la toma metálica.

\section{Parámetros termodinámicos de la adsorción de Cr (VI) y Ni (II)}

De la Tabla 5, se establece que todos los procesos de remoción de $\mathrm{Cr}(\mathrm{VI})$ presentan una adsorción de naturaleza endotérmica debido a un $\Delta \mathrm{H}^{\circ}$ positivo, así se requiere una entrada de energía para provocar la formación de los enlaces entre los iones y los grupos funcionales activos sobre el adsorbente. Además de acuerdo a su valor, para la torta residual de almidón de ñame y plátano se sugiere una quimisorción y una fisisorción sobre el bagazo de palma (Arshadi et al., 2014). Los valores positivos de $\Delta G^{\circ}$ indican que el sistema no es espontaneo para la adsorción de $\mathrm{Cr}(\mathrm{VI})$, el valor negativo sugirió que el proceso con la torta residual de ñame es termodinámicamente factible a $349,15 \mathrm{~K}$, además el hecho de que el $\Delta \mathrm{G}^{\circ}$ aumenta a medida que aumenta la temperatura del sistema para el bagazo de palma hace el proceso energéticamente más favorable. El valor positivo de $\Delta S^{\circ}$, expone la alta afinidad de los iones de $\mathrm{Cr}(\mathrm{VI})$ con los biomateriales y también la probabilidad de algunos cambios estructurales debidos a la formación de enlaces $\mathrm{Cr}$ (VI) con grupos funcionales en la interfaz, indica también que este proceso es reversible (El-Halwany y Mahmoud, 2013).

Tabla 5. Valores de parámetros termodinámicos en (KJ/mol) en la adsorción de $\mathrm{Cr}(\mathrm{VI})$ y Ni (II).

\begin{tabular}{|c|c|c|c|c|c|c|c|}
\hline \multirow{2}{*}{ Biomass } & & \multicolumn{3}{|c|}{$\mathrm{Cr}(\mathrm{VI})$} & \multicolumn{3}{|c|}{$\mathrm{Ni}(\mathrm{II})$} \\
\hline & $\mathrm{T}(\mathrm{K})$ & $\Delta \mathrm{G}^{\circ}$ & $\Delta \mathrm{H}^{\circ}$ & $\Delta \mathrm{S}^{\circ}$ & $\Delta \mathrm{G}^{\circ}$ & $\Delta \mathrm{H}^{\circ}$ & $\Delta \mathrm{S}^{\circ}$ \\
\hline \multirow{3}{*}{ Torta residual de almidón de plátano } & 306,15 & 17,38 & 45,57 & 0,092 & 9,71 & $-8,83$ & $-0,060$ \\
\hline & 328,15 & 15,35 & - & - & 11,05 & - & \\
\hline & 349,15 & 13,42 & - & - & 21,45 & - & \\
\hline \multirow{3}{*}{ Torta residual de almidón de ñame } & 303,15 & 23,97 & 228,56 & 0,675 & 15,77 & 0,232 & $-0,053$ \\
\hline & 328,15 & 7,11 & - & - & 17,09 & - & - \\
\hline & 349,15 & $-7,07$ & - & - & 18,20 & - & - \\
\hline \multirow{3}{*}{ Bagazo de palma } & 303,15 & 30,19 & 3,45 & 0,096 & $-84,29$ & $-107,32$ & $-0,086$ \\
\hline & 328,15 & 32,68 & - & - & $-91,25$ & & \\
\hline & 349,15 & 35,17 & - & - & $-98,20$ & & \\
\hline
\end{tabular}

Del proceso de adsorción de Ni (II) mostrados también en la Tabla 5, se establece que la torta residual de almidón de plátano y el bagazo de palma obedecen a un proceso de naturaleza exotérmica, mientras que sobre la torta residual de ñame es endotérmico. Además, se sugiere una quimisorción para el bagazo de palma, y una fisisorción sobre las tortas residuales confirmando una interacción débil entre ellas y el ion metálico. En la adsorción física las interacciones predominantes son de tipo van der Waals, mientras que en la adsorción química las interacciones semejan enlaces químicos. Los valores positivos de $\Delta G^{\circ}$ sobre las tortas indican la no espontaneidad del proceso y la baja capacidad que tiene para evolucionar por sí mismo, mientras sucede todo lo contrario con el bagazo. La magnitud negativa de $\Delta \mathrm{S}^{\circ}$ representa una disminución de la interacción en la interfaz adsorbente-solución de Ni (II) (Arshadi et al., 2014).

\section{CONCLUSIONES}

Se estudió la adsorción de $\mathrm{Cr}$ (VI) y Ni (II) utilizando residuos agroindustriales como la torta residual de almidón de plátano, torta residual de ñame y bagazo de palma. Se logró una máxima capacidad de remoción de 49,58 $\mathrm{mg} / \mathrm{g}$ para el $\mathrm{Cr}(\mathrm{VI})$ con el bagazo de palma y de $28,004 \mathrm{mg} / \mathrm{g}$ para el $\mathrm{Ni}$ (II) con la torta residual de ñame. Además de acuerdo con el estudio de la termodinámica de adsorción, para el $\mathrm{Cr}(\mathrm{VI})$ usando la torta residual de almidón de ñame y plátano se establece un proceso de quimisorción, y una fisisorción sobre el bagazo de palma, para la remoción de $\mathrm{Ni}$ (II) con el bagazo de palma se sugiere una quimisorción, y una fisisorción sobre las tortas residuales de almidón, confirmando una interacción débil entre estas y el ion níquel cuando se da el proceso de remoción.

\section{AGRADECIMIENTOS}

Los autores agradecen a la Universidad de Cartagena por proporcionar materiales, equipamiento y recurso humanos, requeridos para llevar a cabo el estudio y a COLCIENCIAS por el apoyo financiero a través del Programa de Jóvenes Investigadores.

\section{REFERENCIAS}

Aguayo-Villarreal, I., A. Bonilla-Petriciolet y R. Muñiz-Valencia, Preparation of Activated Carbons from Pecan Nutshell and their Application in the Antagonistic Adsorption of Heavy Metal Ions, doi: 10.1016/j.molliq.2017.01.039, Journal of Molecular Liquids, 230, 686-695 (2017) 
Arshadi, M., M. Amiri y S. Mousavi, Kinetic, Equilibrium and Thermodynamic Investigations of Ni (II), Cd (II), Cu (II) and Co (II) Adsorption on Barley Straw Ash, doi: 10.1016/j.wri.2014.06.001, Water Resources and Industry, 6, 1-17 (2014)

Asuquo, E. y A. Martin, Sorption of Cadmium (II) ion from Aqueous Solution onto Sweet Potato (Ipomoea batatas L.) Peel Adsorbent: Characterisation, Kinetic and Isotherm Studies, doi: 10.1016/j.jece.2016.09.024, Journal of Environmental Chemical Engineering, 4, 4207-4228 (2016)

Chao, P., C. Chang y A. Nieva, Biosorption of Heavy Metals on Citrus maxima Peel, Passion Fruit Shell, and Sugarcane Bagasse in a Fixed-Bed Column, doi: 10.1016/j.jiec.2013.12.027, Journal of Industrial and Engineering Chemistry, 20, 3408-3414 (2014)

Choppala, G., N. Bolan y otros dos autores, The Influence of Biochar and Black Carbon on Reduction and Bioavailability of Chromate in Soils, doi: 10.2134/jeq2011.0145, Journal of Environmental Quality, 41(4) 1175-1184 (2012)

El-Halwany, M. y M. Mahmoud, Kinetics and Thermodynamics of Date Palm Fibers (DPF) as Agricultural Waste Materials, doi: 10.4172/2157-7064.1000194, Journal of Chromatography and Separation Techniques, 4(7) 1-7 (2013)

Ghorbel-Abid, I. y M. Trabelsi-Ayadi, Competitive Adsorption of Heavy Metals on Local Landfill Clay, doi: 10.1016/j.arabjc.2011.02.030, Arabian Journal of Chemistry, 8, 25-31 (2015)

Guiza, S., Biosorption of Heavy Metal from Aqueous Solution Using Cellulosic Waste Orange Peel, doi: 10.1016/j.ecoleng.2016.11.043, Ecological Engineering, 99,134-140 (2017)

Kapur, M. y M. Mondal, Design and Model Parameters Estimation for Fixed-Bed Column Adsorption of Cu(II) and Ni(II) lons Using Magnetized Saw Dust, doi: 10.1080/19443994.2015.1049961, Desalination and Water Treatment, 57, 1219212203 (2016)

Maniglia, B. y D. Tapia-Blácido, Isolation and Characterization of Starch from Babassu Mesocarp, doi: 10.1016/j.foodhyd.2015.11.001, Food Hydrocolloids, 55, 47-55 (2016)

Manirethan, V., K. Raval y otros tres autores, Kinetic and Thermodynamic Studies on the Adsorption of Heavy Metals from Aqueous Solution by Melanin Nanopigment Obtained from Marine Source: Pseudomonas stutzeri, doi: 10.1016/j.jenvman.2018.02.084, Journal of Environmental Management, 214, 315-324 (2018)

Mishra, A., B. Tripathi y A. Rai, Packed-bed Column Biosorption of Chromium (VI) and Nickel (II) onto Fenton Modified Hydrilla Verticillata Dried Biomass, doi: 10.1016/j.ecoenv.2016.06.026, Ecotoxicology and Environmental Safety, 132, 420428 (2016)

Pedrosa, A., O. Herrera y otros cinco autores, Modeling Adsorption of Copper (II), Cobalt (II) and Nickel (II) Metal lons from Aqueous Solution onto a New Carboxylated Sugarcane Bagasse, Part II: Optimization of monocomponent fixed-bed column adsorption, doi: 10.1016/j.jcis.2018.01.068, Journal of Colloid and Interface Science, 516, $431-445$ (2018)

Pradhan, P., A. Arora, y S. Mahajani, Pilot Scale Evaluation of Fuel Pellets Production from Garden Waste Biomass, doi: 10.1016/j.esd.2017.11.005, Energy for Sustainable Development, 43, 1-14 (2018)

Qiu, Q., X. Jiang y otros seis autores, Adsorption of Heavy Metal lons Using Zeolite Materials of Municipal Solid Waste Incineration Fly Ash Modified by Microwave-Assisted Hydrothermal Treatment, doi: 10.1016/j.powtec.2018.05.003, Powder Technology, 335, 156-163 (2018)

Qu, J., X. Meng y otros cuatro autores, Enhanced Removal of Cd (II) from Water Using Sulfur-Functionalized Rice Husk: Characterization, Adsorptive Performance and Mechanism Exploration, doi: 10.1016/j.jclepro.2018.02.208, Journal of Cleaner Production, 183, 880-886 (2018)

Ramos, P., M. Tombini y otros cinco autores, Phenomenological Mathematical Modeling of Heavy Metal Biosorption in Fixed-Bed Columns, doi: 10.1016/j.cej.2017.05.157, Chemical Engineering Journal, 326, 389-400 (2017)

Sreenivas, K., M. Inarkar y otros dos autores, Re-Utilization of Ash Gourd (Benincasa hispida) Peel Waste for Chromium (VI) Biosorption: Equilibrium and Column Studies, doi: 10.1016/j.jece.2014.01.017, Journal of Environmental Chemical Engineering, 2(1), 455-462 (2014)

Tohdee, K. y L. Asadullah, Enhancement of Adsorption Efficiency of Heavy Metal Cu (II) and Zn (II) onto Cationic Surfactant Modified Bentonite, doi: 10.1016/j.jece.2018.04.030, Journal of Environmental Chemical Engineering, 6, 2821-2828 (2018)

Vilvanathan, S. y S. Shanthakumar, Column Adsorption Studies on Nickel and Cobalt Removal from Aqueous Solution Using Native and Biochar form of Tectona grandis, doi: 10.1002/ep.12567, Environmental Progress \& Sustainable Energy, 36, 1030-1038 (2017)

Xie, X., H. Xiong y otros cuatro autores, Preparation Magnetic Cassava Residue Microspheres and its Application for Cu (II) Adsorption, doi: 10.1016/j.jece.2017.05.024, Journal of Environmental Chemical Engineering, 5, 2800-2806 (2017)

Zhang, L., Y. Zeng y Z. Cheng, Removal of Heavy Metal ions Using Chitosan and Modified Chitosan: A review, doi: 10.1016/j.molliq.2015.12.013, Journal of Molecular Liquids, 214, 175-191 (2016) 